International Mathematical Forum, 2, 2007, no. 3, 103 - 108

\title{
The Commutativity in Prime Gamma Rings with Left Derivation
}

\author{
Mustafa AŞCI \\ Gazi University, Science and Art Faculty \\ Department of Mathematics, Ankara, Turkey \\ masci@gazi.edu.tr \\ Şahin CERAN \\ Pamukkale University, Science and Art Faculty \\ Department of Mathematics, Denizli, Turkey \\ sceran@pamukkale.edu.tr
}

\begin{abstract}
Let $\mathrm{M}$ be a $\Gamma-\operatorname{ring}, U$ an ideal of $M$, $\operatorname{char} M \neq 2,3$ and $0 \neq d: M \rightarrow M$ be a left derivation. In this paper we have proved the following results.

(i) If $d(U) \subset U$ and $d^{2}(U) \subset Z$ then $M$ is commutative where $Z=\{c \in$ $M: c \gamma m=m \gamma c: m \in M, \gamma \in \Gamma\}$ is the center of $M$.

(ii) Let $d_{1}, d_{2}$ be non zero left and right derivations of $M$ and $d_{2}(U) \subset U$. If $d_{1} d_{2}(U) \subset Z$ then $M$ is commutative.

Mathematics Subject Classification: Primary 16Y30, 16W25; Secondary $16 \mathrm{U} 80$
\end{abstract}

Keywords: Left derivation, commutativity, prime rings

\section{INTRODUCTION}

The gamma ring is defined in [1] as follows. Let $M$ and $\Gamma$ be additive Abelian groups. If for $a, b, c \in M$ and $\alpha, \beta \in \Gamma$ the following conditions are satisfied.

$\left(\mathrm{G}_{1}\right) a \beta b \in M$

$\left(\mathrm{G}_{2}\right)(a+b) \alpha c=a \alpha c+b \alpha c, a(\alpha+\beta) b=a \alpha b+a \beta b, a \alpha(b+c)=a \alpha b+a \alpha c$

$\left(\mathrm{G}_{3}\right)(a \alpha b) \beta c=a \alpha(b \beta c)$

Then $M$ is called a $\Gamma$-ring (in the sense of Barnes). Every ring is a $\Gamma$-ring. A right (left) ideal of a $\Gamma$-ring $M$ is an additive subgroup $U$ of $M$ such that $U \Gamma M \subset U(M \Gamma U \subset U)$. If $U$ is both a right and left ideal, then we say that $U$ is an ideal of $M$. If the following conditions hold for a gamma ring $M$ then $M$ 
is called a prime gamma ring [2]. For $a, b \in M$

$$
a \Gamma M \Gamma b=0 \Rightarrow a=0 \text { or } b=0
$$

In this paper we will prove some results in gamma rings which are known in ring theory. Throughout this paper $M$ will be a prime $\Gamma-\operatorname{ring}, Z$ will be the center of $M$.

Let $M$ be a $\Gamma$-ring and $D: M \rightarrow M$ be an additive map. $D$ is called a left derivation if for all $x, y \in M, \alpha \in \Gamma$

$$
D(x \alpha y)=x \alpha D(y)+y \alpha D(x)
$$

A right derivation is defined similarly. A $\Gamma-\operatorname{ring} M$ is called commutative if $x \alpha y=y \alpha x$ for any $x, y \in M$ and $\alpha \in \Gamma$. An element $m \in M$ is called a right nonzero divisor if $x \alpha m=0$ implies $x=0$ for any $\alpha \in \Gamma$. A left nonzero divisor is defined similarly.

\section{Main Results}

Lemma 1. Let $U$ be a non-zero right ideal of $M$ and $d: M \rightarrow M$ left derivation (right derivation).

(i) If $d(U)=0$ then $d=0$

(ii) Let $U$ be a non-zero ideal of $M$ and $d: M \rightarrow M$ right derivation, $d(M)$ left non-zero divisor. If $a \Gamma d(U)=0$ then $a=0$

(iii) Let $U$ be a non-zero ideal of $M$ and $d: M \rightarrow M$ left derivation, $D(M)$ right non-zero divisor. If $d(U) \Gamma a=0$ then $a=0$

(iv) Let $U$ be a non-zero ideal of $M$ and charM $\neq 2, d: M \rightarrow M$ right derivation. If $d^{2}(U)=0$ then $d=0$

(v) Let $U$ be a non-zero ideal of $M$, char $M=2, d_{1}: M \rightarrow M$ left derivation and $d_{2}: M \rightarrow M$ right derivation. If $d_{2}(U) \subset U$ and $d_{1} d_{2}(U)=0$ then $d_{1}=0$ or $d_{2}=0$

Proof. (i) For any $u \in U, \alpha \in \Gamma$ and $m \in M$ we have

$$
\begin{aligned}
0 & =d(u \alpha m)=u \alpha d(m)+\operatorname{m\alpha d}(u) \\
& ==u \alpha d(m)
\end{aligned}
$$

That is, $U \Gamma d(M)=0$. This implies that $d(M)=0$, by [[4] Lemma 2 (ii)] and so $d=0$

(ii) Let $\alpha, \beta \in \Gamma, u \in U$ and $m \in M$. Then

$$
\begin{aligned}
0 & =a \gamma d(u \beta m) \\
& =a \gamma d(u) \beta m+a \gamma d(m) \beta u \\
& =a \gamma d(m) \beta u
\end{aligned}
$$


is obtained. That is, $a \Gamma d(M) \Gamma U=0$ Hence $a \Gamma d(M)=0$ by [[4] Lemma 2 (ii)] and

$$
\begin{aligned}
0 & =a \Gamma d(m \gamma) d^{-1}(m) \\
& =a \Gamma d(m) \gamma d^{-1}(m)+a \Gamma m \Gamma m \\
& =a \Gamma M \Gamma M
\end{aligned}
$$

[[4] Lemma 2 (ii)] $a=0$

(iii) Let $\alpha, \beta \in \Gamma, u \in U$ and $m \in M$. Then

$$
\begin{aligned}
0 & =d(u \beta m) \gamma a \\
& =u \beta d(m) \gamma a+m \beta d(u) \gamma a \\
& =u \beta d(m) \gamma a
\end{aligned}
$$

is obtained. That is, $U \Gamma d(M) \Gamma a=0$ hence $d(M) \Gamma a=0$, [[4] by Lemma 2 (ii)] and

$$
d\left(m \gamma d^{-1}(m)\right) \Gamma a=m \gamma m \Gamma a+d^{-1}(m) \gamma d(m) \Gamma a=>M \Gamma M \Gamma a=0
$$

[[4] Lemma 2 (ii)], $a=0$

(iv) Let $\operatorname{char} M \neq 2$ and $d^{2}(U)=0$. For any $u \in U$ and $\alpha \in \Gamma$ we have $0=d^{2}(u \alpha u)=d(u) \alpha d(u)$. Therefore, $d(U) \Gamma d(U)=0$ and so $d(U)=0$ by (ii). Hence $d=0$ by (i)

(v) If $d_{1} d_{2}(U)=0$ and $d_{2}(U) \subset U$ then for all $u, v \in U$ and $\alpha \in \Gamma$

$$
\begin{aligned}
0 & =d_{1} d_{2}(u \alpha v)=d_{1}\left(d_{2}(u) \alpha v+d_{2}(v) \alpha u\right) \\
& =d_{2}(u) \alpha d_{1}(v)+v \alpha d_{1} d_{2}(v)+d_{2}(v) \alpha d_{1}(u)+u \alpha d_{1} d_{2}(v) \\
& =d_{2}(u) \alpha d_{1}(v)+d_{2}(v) \alpha d_{1}(u)
\end{aligned}
$$

If we replace $u$ by $d_{2}(u)$ in the last equation we get $d_{2}^{2}(U) \Gamma d_{1}(U)=0$ By (ii) and (iii), we obtain $d_{1}=0$ or $d_{2}=0$

In the rest of this paper $U$ will be a nonzero ideal of $M$.

Lemma 2. Let $d$ be a non-zero right derivation of $M$ and charM $\neq 2$. If $d(U) \subset Z$ then $M$ is commutative.

Proof. For all $u \in U, y \in M, z \in Z$ and $\gamma \in \Gamma$

$$
\begin{aligned}
0 & =[d(u \gamma z), y]_{\gamma} \\
& =[d(u) \gamma z+d(z) \gamma u, y]_{\gamma} \\
& =d(z) \gamma[u, y]_{\gamma}
\end{aligned}
$$

This implies that

$$
[U, M]_{\gamma}=0 \text { or } d(Z)=0
$$

by [[4] Lemma 1 (vi)] for all $\gamma \in \Gamma$. If $d(Z)=0$ then $d^{2}(U)=0$, which implies that $d=0$ by [[4] Lemma 1 (iv)]. This is a contradiction. Thus $U \subset Z$ is obtained and so $M$ is commutative by [[4] Lemma 2 (i)]. 
Theorem 3. Let $d$ be a non-zero right derivation of $M$ and $\operatorname{char} M \neq 2,3$. If $d^{2}(U) \subset Z$ and $d(U) \subset U$ then $M$ is commutative.

Proof. For any $u \in U, \gamma, \beta \in \Gamma$ and $y \in M$ we have,

$$
\begin{aligned}
0 & =\left[d^{2}(d(u) \gamma d(u)), y\right]_{\beta} \\
& =2\left[d\left(d^{2}(u) \gamma d(u)\right), y\right]_{\beta} \\
& =2\left[d^{3}(u) \gamma d(u)+d^{2}(u) \gamma d^{2}(u), y\right]_{\beta} \\
& =2 d^{3}(u) \gamma[d(u), y]_{\beta}
\end{aligned}
$$

and so by [[4]Lemma 1(v)]

$$
d^{3}(u)=0 \quad \text { or } \quad d(u) \in Z
$$

is obtained. If $d^{3}(U)=0$ then

$$
\begin{aligned}
0 & =\left[d^{2}(u \beta d(u)), y\right]_{\gamma} \\
& =\left[d\left(d(u) \beta d(u)+d^{2}(u) \beta u\right), y\right]_{\gamma} \\
& =\left[2 d^{2}(u) \beta d(u)+d^{3}(u) \beta u+d(u) \beta d^{2}(u), y\right]_{\gamma} \\
& =2 d^{2}(u) \beta[d(u), y]_{\gamma}+[d(u), y]_{\gamma} \beta d^{2}(u) \\
& =3 d^{2}(u) \beta[d(u), y]_{\gamma}
\end{aligned}
$$

Considering $\operatorname{char} M \neq 3$ and using [[4] Lemma 1 (v)] we get for all $u \in U$

$$
d^{2}(u)=0 \text { or } d(u) \in Z
$$

the sets $K=\left\{u \in U: d^{2}(u)=0\right\}$ and $L=\{u \in U: d(u) \in Z\}$ are additive subgroups of $U$ and $U=K \cup L$ If $U=K$ then $d=0$ by Lemma 1 (iv). This is a contradiction. Hence we have $U=L$ by Brauer trick and so $M$ is commutative by Lemma 2 .

Lemma 4. Let $0 \neq d_{1}: M \rightarrow M$ left derivation and $0 \neq d_{2}: M \rightarrow M$ right derivation such that $d_{1} d_{2}(U) \subset Z$ and $d_{2}(U) \subset U$, charM $\neq 2,3$. If $d_{1} d_{2}^{2}(U)=0$ then $M$ is commutative.

Proof. For any $u \in U$ and $\gamma \in \Gamma$ we have for all $y \in M$.

$$
\begin{aligned}
0 & =\left[d_{1}\left(d_{2}\left(d_{2}(u) \beta d_{2}(u)\right)\right), y\right]_{\gamma} \\
& =2\left[d_{1}\left(d_{2}^{2}(u) \beta d_{2}(u)\right), y\right]_{\gamma} \\
& =2\left[d_{2}^{2}(u) \beta d_{1} d_{2}(u)+d_{2}(u) \beta d_{1} d_{2}^{2}(u), y\right]_{\gamma} \\
& =2\left[d_{2}^{2}(u) \beta d_{1} d_{2}(u), y\right]_{\gamma}
\end{aligned}
$$

Since $\operatorname{char} M \neq 2$, if we use [lemma 1(iv)] we get

$$
d_{1} d_{2}(u)=0 \text { or } d_{2}^{2}(u) \in Z
$$

Let $K=\left\{u \in U: d_{1} d_{2}(u)=0\right\}$ and $L=\left\{u \in U: d_{2}^{2}(u) \in Z\right\}$. In this case $K$ and $L$ are subgroups of $U$ and $U=K \cup L$. If $U=K$ then $d_{1}=0$ or $d_{2}=0$ by Lemma $1(\mathrm{v})$. This is a contradiction. Therefore $U=L$ by Brauer trick, which implies that $M$ is commutative by Theorem 1. 
Lemma 5. Let $0 \neq d_{1}: M \rightarrow M$ left derivation and $0 \neq d_{2}: M \rightarrow M$ right derivation such that $d_{1} d_{2}(U) \subset Z, d_{2}(U) \subset U, \operatorname{char} M \neq 2$ and $a \in M$. If $\left[d_{1}(U), a\right]_{\gamma}=0$ then $\left[d_{2}(U), a\right]_{\gamma}=0$ for all $\gamma \in \Gamma$.

Proof. For any $x \in U$ and $\gamma \in \Gamma$ we have

$$
\begin{aligned}
0 & =\left[d_{1} d_{2}(x) \gamma d_{2}(x), a\right]_{\gamma} \\
& =2\left[d_{2}(x) \gamma d_{1} d_{2}(x), a\right]_{\gamma} \\
& =2\left[d_{2}(x), a\right]_{\gamma} \gamma d_{1} d_{2}(x)
\end{aligned}
$$

By [[4] Lemma 1 (iv)] for all $x \in U, \gamma \in \Gamma$

$$
d_{1} d_{2}(x)=0 \quad \text { or }\left[d_{2}(x), a\right]_{\gamma}=0
$$

is obtained. Let $K=\left\{x \in U: d_{1} d_{2}(x)=0\right\}$ and $L=\left\{x \in U:\left[d_{2}(x), a\right]_{\gamma}=0\right\}$. Then $K$ and $L$ are subgroups of $U$ and $U=K \cup L$. Since $d_{1} \neq 0$ and $d_{2} \neq 0$, $U$ can not equal to $K$ by Lemma $1(\mathrm{v})$. Hence $U=L$ by Brauer trick. That is $\left[d_{2}(U), a\right]_{\gamma}=0$ for all $\gamma \in \Gamma$, is obtained.

Lemma 6. Let $d_{1}$ be non-zero left derivation of $M$ and $d_{2}$ be non-zero right derivation of $M$ such that $d_{1} d_{2}(U) \subset Z, d_{2}(U) \subset U$ and $a \in M$. If $\left[d_{1}(U), a\right]_{\gamma}=$ 0 for all $\gamma \in \Gamma$ then $a \in Z$.

Proof. By Lemma 4 we have $\left[d_{2}(U), a\right]_{\gamma}=0$ for all $\gamma \in \Gamma$. Hence for all $x \in U$ and $\gamma \in \Gamma$

$$
\begin{aligned}
0 & =\left[d_{1} d_{2}(x \gamma x), a\right]_{\gamma} \\
& =2\left[d_{1}\left(d_{2}(x) \gamma x\right), a\right]_{\gamma} \\
& =2\left[d_{2}(x) \gamma d_{1}(x)+x \gamma d_{1} d_{2}(x), a\right]_{\gamma} \\
& =2\left[x \gamma d_{1} d_{2}(x), a\right]_{\gamma}
\end{aligned}
$$

And so

$$
d_{1} d_{2}(x)=0 \text { or }[x, a]_{\gamma}=0
$$

[[4] Lemma 1 (iv)]. If we apply to (2.5) Brauer trick and use Lemma 1 (v) we have $a \in Z$.

Theorem 7. Let $d_{1}$ be non-zero left derivation of $M$ and $d_{1} d_{2}(U) \subset Z$ and $d_{2}(U) \subset U$ and $a \in M$, char $M \neq 2$, 3. If $\left[d_{1}(U), a\right]_{\gamma} \in Z$ for all $\gamma \in \Gamma$ then $a \in Z$.

Proof. If $Z=0 d_{1} d_{2}(U)=0$ and so $d_{1}=0$ or $d_{2}=0$ by Lemma 1 . It is a contradiction. Hence we can assume that $Z \neq 0$. For any $x \in U, z \in Z$ and $\gamma, \beta \in \Gamma$ we have

$$
\begin{aligned}
Z & \ni\left[d_{1}(x \beta z), a\right]_{\gamma}=\left[x \beta d_{1}(z)+z \beta d_{1}(x), a\right]_{\gamma} \\
& =[x, a]_{\gamma} \beta d_{1}(z)+z \beta\left[d_{1}(x), a\right]_{\gamma}
\end{aligned}
$$

That is, for all $\gamma, \beta \in \Gamma$

$$
[U, a]_{\gamma} \beta d_{1}(z) \subset Z
$$


By [[4] Lemma $1(\mathrm{v})]$ and (2.6) we obtain for all $\gamma \in \Gamma$

$$
[U, a]_{\gamma} \subset Z \text { or } d_{1}(z)=0
$$

If $d_{1}(z) \neq 0$ then $[U, a]_{\gamma} \subset Z$, for all $\gamma \in \Gamma$. Now, for any $m \in M, \beta \in \Gamma$, we have $\left[[U, a]_{\gamma}, m\right]_{\beta}=0$. Let us take $u \gamma u$ instead of $u$ in the last equation. Then we get,

$$
\begin{aligned}
0 & =\left[[u \gamma u, a]_{\gamma}, m\right]_{\beta} \\
& =\left[u \gamma[u, a]_{\gamma}+[u, a]_{\gamma} \gamma u, m\right]_{\beta} \\
& =2\left[u \gamma[u, a]_{\gamma}, m\right]_{\beta}
\end{aligned}
$$

Since $M$ is a prime gamma ring, then for all $m \in M, \gamma, \beta \in \Gamma, u \in U$ we have

$$
[u, m]_{\beta} \gamma[u, a]_{\gamma}=0
$$

This implies that, [[4] by Lemma 1 (iv)] for all $m \in M, \beta, \gamma \in \Gamma$

$$
[u, m]_{\beta}=0 \text { or }[u, a]_{\gamma}=0
$$

That is, for all $\gamma \in \Gamma$

$$
[U, a]_{\gamma}=0
$$

If we consider Lemma 5 and (2.9) we get $a \in Z$.

If $d_{1}(z)=0$ then $d_{1} d_{1} d_{2}(U)=0$. Thus for any $x \in U$ and $\gamma \in \Gamma$ we have

$$
\begin{aligned}
0 & =d_{1} d_{1} d_{2}\left(d_{2}(x) \gamma d_{2}(x)\right) \\
& =d_{1} d_{1}\left(2 d_{2}^{2}(x) \gamma d_{2}(x)\right) \\
& =2 d_{1}\left(d_{2}^{2}(x) \gamma d_{1} d_{2}(x)+d_{2}(x) \gamma d_{1} d_{2}^{2}(x)\right) \\
& =4 d_{1} d_{2}(x) \gamma d_{1} d_{2}^{2}(x)
\end{aligned}
$$

and so for all $x \in U$

$$
d_{1} d_{2}(x)=0 \text { or } d_{1} d_{2}^{2}(x)=0
$$

If we say that $K=\left\{x \in U: d_{1} d_{2}(x)=0\right\}$ and $L=\left\{x \in U: d_{1} d_{2}^{2}(x)=0\right\}$ then $K$ and $L$ are subgroups of $U$ and $U=K \cup L$. Since $d_{1} \neq 0$ and $d_{2} \neq 0$, we have $U \neq K$ by Lemma 1. Thus if we consider Brauer trick we get $U=L$. Thus it $d_{1} d_{2}^{2}(U)=0$. Now, $d_{1} d_{2}^{2}(U)=0, d_{1} d_{2}(U) \subset Z, d_{2}(U) \subset U$ and $\operatorname{char} M \neq 2,3$ implies that $M$ is commutative by Lemma 3 and $a \in Z$.

\section{REFERENCES}

[1] Barnes W. E., On the Г-rings of Nobusawa, Pacific J. of Math.Vol. 18, No 3 , (1966).

[2] Kyum S. , On prime gamma rings, Pacific J. of Math.Vol. 25, No 1 , (1978).

[3] Feng Jie Jing, On derivations of $\Gamma$-rings; Qu fu shifon Daxue Xuebeo Ziran Kexue Ban 13 No 4, 159 - 161 (1987)

[4] Soytürk M., The commutativity in prime gamma rings with derivation, Tr. J.Of Math.Vol. 18. 149 - 155, (1994).

Received: May 30, 2006 